\title{
Theoretical observation of optical Fano resonance at Gratting nano structure
}

\author{
Maryam HAJILARI ${ }^{1}$, Mahdi ZAVVARI ${ }^{2 *}$ \\ ${ }^{1}$ Department of Electrical Engineering, Ahar branch, Islamic Azad University, Ahar, Iran. \\ ${ }^{2}$ Department of Electrical Engineering, Urmia branch, Islamic Azad University, Urmia, Iran. \\ *Corresponding Author: m.zavvari@iaurmia.ac.ir
}

\begin{abstract}
Recently, Fano resonances have been studied in nano particles, plasmonics, photonic crystals and electromagnetic metamaterials .In this study, we studied the Fano resonance in individual semiconductor and investigated the effects on gratting.

In our studies, we introduced a simple semiconductor nano-structure and described the simulations and analysis of optical Fano resonance in scattering and absorption spectra of structure and investigated experimentally it as a function of different thickness, change the width and radius of semiconductor and at different wavelengths at periodic conditions and angle of incidence equal $0^{\circ}$. According to the results, Fano resonances are observed in the mentioned structures and they are depend on angle of incidence, width and radius of structure, wavelengths.
\end{abstract}

Keywords: Fano resonance, scattering, reflection, transmission, absorption, refractive index, angle of incidence, Gratting.

\section{INTRODUCTION}

Resonances such as mechanical, acoustic and electromagnetic are one of the wide variety of quantum and classical systems. In this research optical Fano resonance with an asymmetric characteristic of quantum systems interaction has been studied in individual semiconductor nanostructure. Generally, we can said that resonances mostly regulated by veriaty of geometric parameters.

Fano resonances with an asymmetric characteristic can be observed by light scattering and reflection spectrum of the optical systems. They are results of direct and indirect interference between paths.

In recent years, a wide variety of applications used for surface plasmon. Of course advance of this science depends on techniques such as optical lithographeis and saveing of optic datas. The growing desire in this branch of science can be give to break down and desending limit of the light diffraction and extenuant order of wavelengths. This knowledge can be used in electronic and optical circuits, separately so that with simultaneous propagation in each of these basis and plasmonic waves don $t$ arrive disturbance to transmission of each waves datas. This makes that it can be transmitted data with high speed in integrated circuits.

Researchers could give some good results about Optical Fano resonance of an individual semiconductor nanostructure at 2014. They gave some results about near and far-field characterization of Fano resonances in a high-index semiconductor nano structure, Theoretical study of the Fano resonance observed from a Si nano stripe under different illumination conditions, Measurement and simulation of the scattering spectra of Si nanostripes, Measured absorption spectra from a Si nanostripe for different illumination conditions.

We can present some others searches about these projects such as Fano resonances in quasi-onedimensional electron waveguides, Symmetry breaking in plasmonic nano cavities: subradiant LSPR sensing and a tunable Fano resonance, The Fano resonance in plasmonic nano structures and 
metamaterials, Removing a wedge from a metallic nano disk reveals a Fano resonance, Nanorod orientation dependence of tunable Fano resonance in plasmonic nanorod heptamers, Optical Fano resonance of an individual semiconductor nano structure [1-6].

\section{Materials and method}

In this paper we report optical Fano resonance in new semiconductor nano structure and investigate line shape of them in different condition, we present a gratting of nano-structure that include three materials : Air , Al , Si . You can see figure 1:
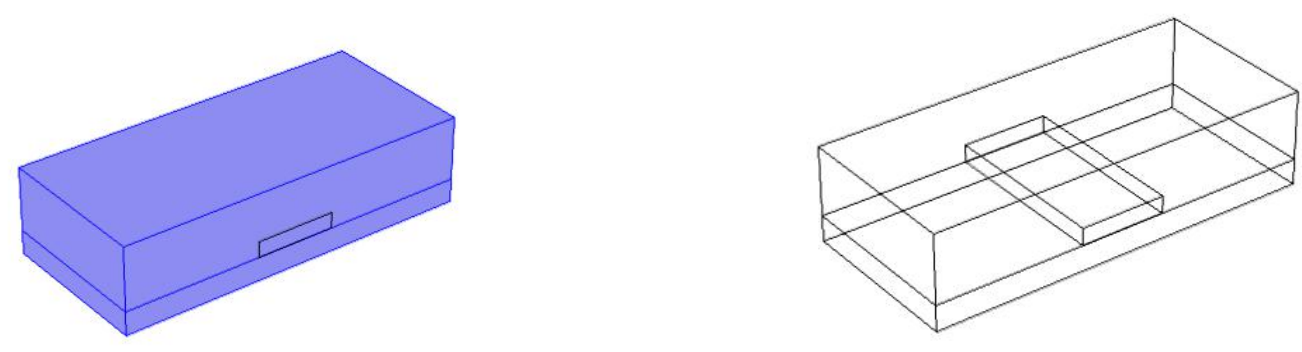

Fig 1 : Gratting nano structure with three layers Air, Al, Si

We use an individual semiconductor nano structure and simulate scattering and absorption for observing the optical Fano resonance .Scattering curve has been obtained by scattering parameters. And for absorption curve, we should have reflection and transmission. All of these have been computed by Comsol software and use Maxwell equations and by using electromagnetic waves at frequency domain and optic waves.Generally, survayes illustrate that the optical Fano resonance depends on some parameters such as :

Refractive index, Real part $=\mathrm{n}$, Refractive index, Imaginary part $=\mathrm{k}$, Relative permittivity $=\varepsilon_{r}$, Relative permeability $=\mu_{r}$, Angle of incidence $=\alpha$, Variation of width, height, radius $=\mathrm{W}, \mathrm{H}$, $\mathrm{R}$, Variation of wavelength $=\mathrm{w}$ or Lambda.

Fig. 1 show 3 dimensions view of the gratting structure, 3 layers include of Air and $\mathrm{Si}$ and nanostripe of $\mathrm{Al}$ between them at the electric field and TE mode. Thickness of air layer is $150 \mathrm{~nm}$ and width of it equal $450 \mathrm{~nm}$, thickness of Si layer is $50 \mathrm{~nm}$ and width of Si equal $400 \mathrm{~nm}$ and thickness of Al layer is $40 \mathrm{~nm}$ and width of it equal $100 \mathrm{~nm}$. we investigate Fano resonance at TE mode and assume periodic conditions. Then we compare line shape of optic Fano resonance at scattering and absorption curve in different condition of instructure. Here, we shoud obtain absorption and scattering for different wavelengths and then we can observe Fano resonance at some wavelengths at electric field.

\section{Results and Discussion}

Optical Fano resonance property of the nanostructure was characterized by absorption or scattering spectral analysis system. Fig 2 shows the results of them at the Fig 1 nano instructure in periodic conditions. 


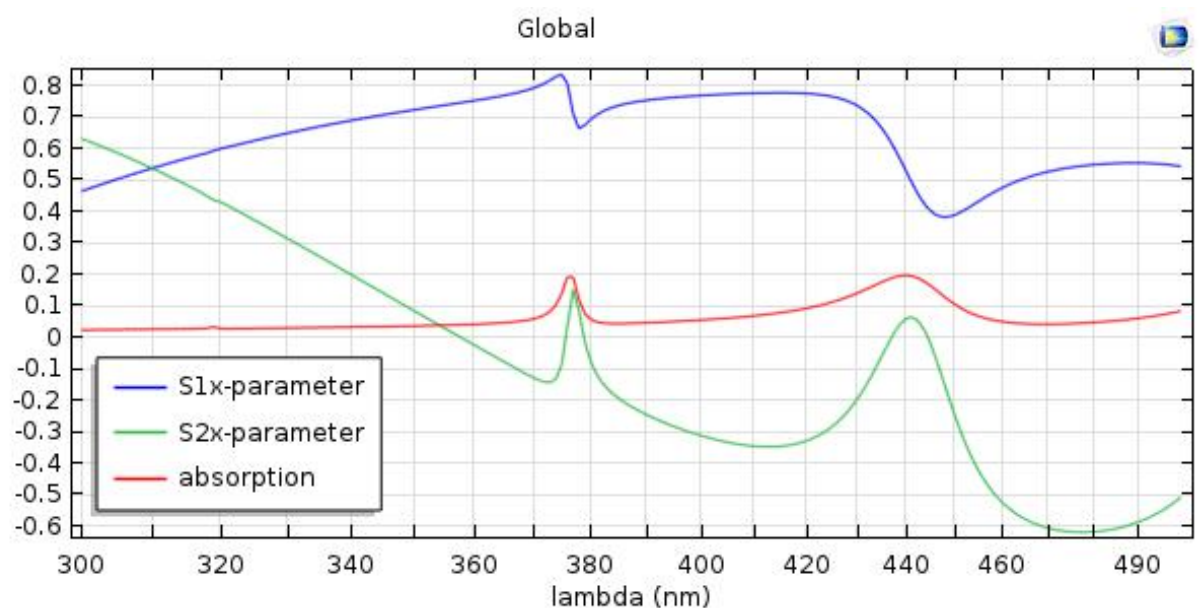

Fig 2 : The results of absorption and scattering at grating nano structure in electric field and TE mode

As shown as Fig 2 , we have 2 absorption and so, 2 Fano resonances at scattering spectrum that happen at $380 \mathrm{~nm}$ and $440 \mathrm{~nm}$ of wavelengths.In fact, Fano resonance is an asymmetric characteristic of scattering curve when we have absorption there. Fig 3 shows the effects of Fano resonance at electric field.

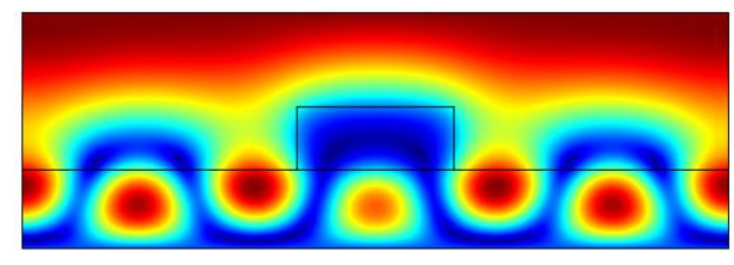

(a)

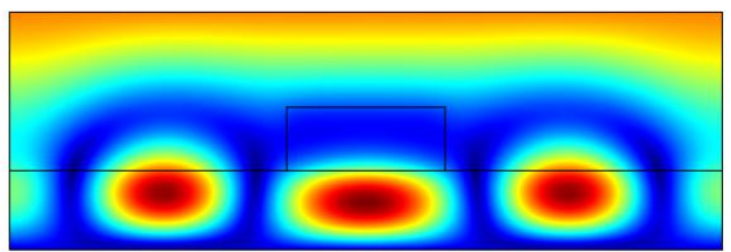

(b)

Fig 3 : (a) The effects of Fano resonance at $380 \mathrm{~nm}$, (b) The effects of Fano resonance at $440 \mathrm{~nm}$

Then we change the gratting nano structure to Fig 4. In this instructure we change the thickness of Al layer to $20 \mathrm{~nm}$ and Si layer to $80 \mathrm{~nm}$ at the same conditions.

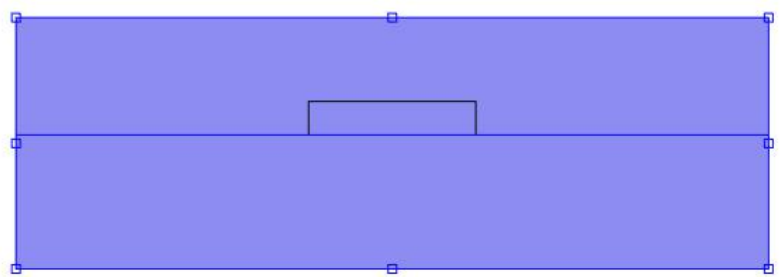

Fig 4 : the changes of nano structure, the thickness of Al layer $20 \mathrm{~nm}$ and Si layer $80 \mathrm{~nm}$.

We can achieve new results about Fano resonances at different conditions as shown as Fig. In these conditions we have more absorption and Fano resonances at scattering spectrum. In fact, we can observe three absorption at 4 different wavelengths and so we can observe optic Fano resonances at these wavelengths. 


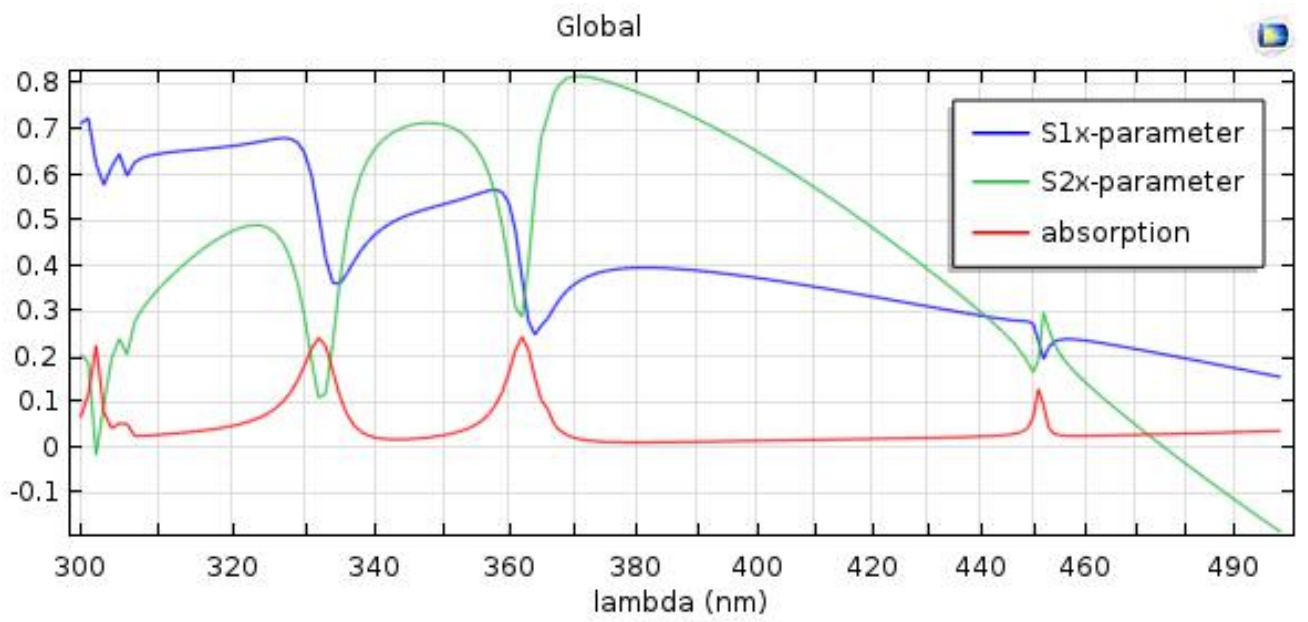

Fig 5 : The results of absorption and scattering at TE mode and the thickness of Al layer $20 \mathrm{~nm}$ and Si layer $80 \mathrm{~nm}$, periodic conditions.

Fig 5 illustrates the properties of the Fano resonance in $\mathrm{Al}$ nano stripes with realistic material dispersion are studied by experiment and simulation. From simulations, It was determined that an $\mathrm{Al}$ stripe with a width of $450 \mathrm{~nm}$ and a thickness of $20 \mathrm{~nm}$ supports a resonance at 4 a wavelengths of $301 \mathrm{~nm}, 332 \mathrm{~nm}, 362 \mathrm{~nm}, 453 \mathrm{~nm}$.

Furthermore, we obtained some results about the effects of optic Fano resonances at electric field at different wavelengths in periodic conditions. Fig 6 shows the results of Fano resonances at electric field in TE mode.

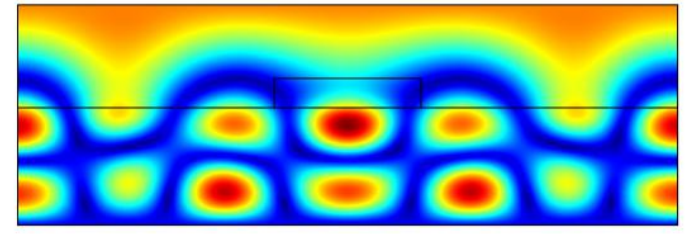

(a)

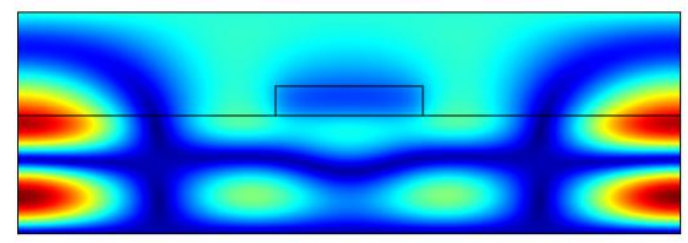

(c)

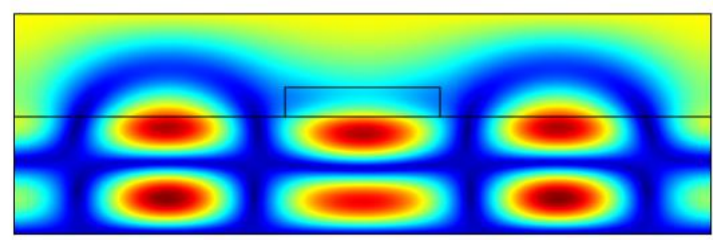

(b)

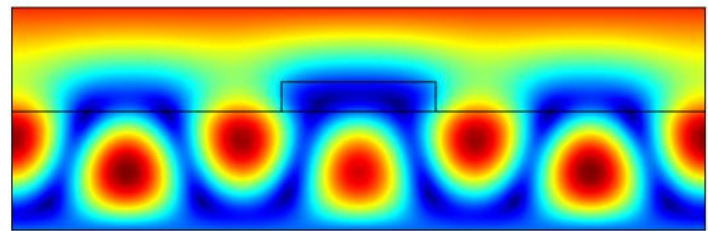

(d)

Fig 6 : (a) Fano resonance at $301 \mathrm{~nm}$, (b) Fano resonance at $332 \mathrm{~nm}$, (c) Fano resonance at $362 \mathrm{~nm}$, (d) Fano resonance at $453 \mathrm{~nm}$ when the thickness of Al layer is $20 \mathrm{~nm}$ and Si layer is 80 $\mathrm{nm}$, periodic conditions.

\section{Conclusions}

Our study theoretically analisis and experimentally demonstrates that optic Fano resonance occure in the scattering spectra of specific gratting nano structure geometry with 3 materials include Air , $\mathrm{Al}$, Si. The changes in the Fano line shape that result for different conditions and stripe sizes. Here 
we use metal nano stripe and present the efects of semiconductor on Fano resonance. As shown as figures, we can conclude that resize of nano stripe and semiconductor have effects on the results of absorption and scattering and so, have effects on optic Fano resonance. At first conditions and size of geometry we have absorption at 2 different wavelengths and assymetric characteristic on scattering spectra and after resize of geometry with different size of Al nano stripe and Si layer observed 4 different wavelengths for absorption and scattering results. In fact, the changes of geometry has direct effects on optic Fano resonances.

\section{References}

1. Tekman E, Bagwell PF. Fano resonances in quasi-one-dimensional electron waveguides. Phys Rev B Condens Matter. 1993;48:2553-9.

2. Hao F, Sonnefraud Y, Van Dorpe P, Maier SA, Halas NJ, Nordlander P. Symmetry breaking in plasmonic nanocavities: subradiant LSPR sensing and a tunable Fano resonance. Nano Lett. 2008;8:3983-8.

3. Luk'yanchuk B, Zheludev NI, Maier SA, Halas NJ, Nordlander P, Giessen H, et al. The Fano resonance in plasmonic nanostructures and metamaterials. Nat Mater. 2010;9:707-15.

4. Fang Z, Cai J, Yan Z, Nordlander P, Halas NJ, Zhu X. Removing a wedge from a metallic nanodisk reveals a fano resonance. Nano Lett. 2011;11:4475-9.

5. Tamma VA, Cui Y, Zhou J, Park W. Nanorod orientation dependence of tunable Fano resonance in plasmonic nanorod heptamers. Nanoscale. 2013;5:1592-602.

6. Fan P, Yu Z, Fan S, Brongersma ML. Optical Fano resonance of an individual semiconductor nanostructure. Nat Mater. 2014;13:471-5. 